\title{
Screening of genes associated with inflammatory responses in the endolymphatic sac reveals underlying mechanisms for autoimmune inner ear diseases
}

\author{
JUHONG ZHANG $^{1,2}$, NA WANG ${ }^{1}$ and ANTING XU ${ }^{1,3}$ \\ ${ }^{1}$ Department of Otolaryngology, Head and Neck Surgery, The Second Hospital of Shandong University, \\ Jinan, Shandong 250033; ${ }^{2}$ Department of Otolaryngology, Shanghai Jiao Tong University, \\ Affiliated to Sixth People's Hospital South Campus, Shanghai 201411; ${ }^{3}$ Department of Otolaryngology, \\ Affiliated Tenth People's Hospital of Tongji University, Shanghai 200072, P.R. China
}

Received December 25, 2017; Accepted June 1, 2018

DOI: $10.3892 /$ etm.2018.6479

\begin{abstract}
The current study analyzed gene expression profiles of the endolymphatic sac (ES) in rats and identified expressed genes, present in the human and rat ES, to reveal key hubs for inflammatory responses. Microarray data (accession no. E-MEXP-3022) were obtained from the European Bioinformatics Institute database, including three biological replicates of ES plus dura tissues and three replicates of pure dura tissues form rats. Differentially expressed genes (DEGs) were screened using the Linear Model for Microarray data method and a protein-protein interaction (PPI) network was constructed using data from the Search Tool for the Retrieval of Interacting Genes/Proteins database followed by a module analysis via Clustering with Overlapping Neighborhood Expansion. Function enrichment analysis was performed using the Database for Annotation, Visualization and Integrated Discovery online tool. A total of 612 DEGs were identified, including 396 upregulated and 216 downregulated genes. Gene ontology term enrichment analysis indicated DEGs were associated with cell adhesion, including $\alpha 5$-integrin (Itga1) and secreted phosphoprotein 1 (Spp1); T cell co-stimulation, including $\mathrm{C}-\mathrm{C}$ chemokine ligand $(\mathrm{Ccl}) 21$ and $\mathrm{Ccl19}$; and the toll-like receptor signaling pathway, including toll-like receptor (Tlr)2, Tlr7 and Tlr8. These conclusions were supported by Kyoto Encyclopedia of Genes and Genomes pathway analyses revealing extracellular matrix-receptor interaction, including Itga1 and Spp1; leukocyte transendothelial migration, includingclaudin-4 (Cldn4); and malaria, including Tlr2. The hub roles of Itga1, Cd24 and Spp1 were
\end{abstract}

Correspondence to: Dr Anting Xu, Department of Otolaryngology, Head and Neck Surgery, The Second Hospital of Shandong University, 247 Beiyuan Street, Jinan, Shandong 250033, P.R. China E-mail: antingxu@sdu.edu.cn

Key words: endolymphatic sac, hearing loss, inflammation, cell adhesion revealed by calculating three topological properties of the PPI network. Ccl21, Ccl19 and Cldn4 were demonstrated to be crucial following significant module analysis according to the corresponding threshold, which revealed they were enriched in inflammation pathways. Tlr7, Tlr2, granzyme $\mathrm{m}$ and Tlr8 were common genes associated with inflammatory responses in rat and human ES. In conclusion, abnormal expression of the aforementioned inflammation-associated genes may be associated with the development of autoimmune inner ear diseases.

\section{Introduction}

Autoimmune inner ear disease (AIED) is one of the few reversible causes of rapidly progressive, bilateral sensorineural hearing loss (SNHL) over a period ranging from weeks to months (1). It has been reported that AIED has a predilection to occur in young adults, but variable prevalence is also observed during childhood (4-30\%) (2,3). SNHL is frequently accompanied by tinnitus, persistent vertigo and increased depression, which disrupt activities and personal relationships by severely affecting the quality of life $(4,5)$.

Currently, corticosteroids are a cornerstone in the treatment of AIED; however, a successful reduction of symptoms and the preservation of hearing can only be achieved in $~ 60 \%$ of patients following timely corticosteroid administration (6). In addition, prolonged use of high-dose corticosteroids (>4 weeks) carries risk of serious adverse events, including hyperglycemia and weight gain (7). In steroid-dependent patients, hearing immediately worsens if the dose declines (8). The development of novel treatment strategies is crucial for the effective management of the disease.

Although the pathogenesis is not fully understood, celland/or humoral-mediated immune injuries serve important roles in the development of AIED (9). When a foreign antigen enters the inner ear, it is first processed by immunocompetent cells present in and around the endolymphatic sac (ES) (10). These immunocompetent cells secrete various cytokines, including interleukin (IL)-1 $\beta$ and tumor necrosis factor (TNF)- $\alpha$, which recruit inflammatory cells from the systemic 
circulation to the cochlea, further amplifying the immune response and deteriorating inner ear damages $(9,11,12)$. Several TNF- $\alpha$ (13) or IL-1 $\beta$ (14) antagonists have been suggested as potential agents for treating patients with AIED with no response to corticosteroid treatment. Alternative medicines for AIED remain rare and further investigations into the crucial genes for inflammatory response in ES are necessary.

Previously, studies analyzed the global gene expression profile of the ES in rats (15) and humans $(16,17)$. In comparison with adjacent dura tissues, several inflammatory response-associated genes were identified, including macrophage migration inhibitory factor, small inducible cytokine subfamily e, member 1 , the $\mathrm{C}-\mathrm{C}$ chemokine ligand $(\mathrm{Ccl}) 21 \mathrm{~b}$ (serine) and toll-like receptor (Tlr)7 $(15,16)$. In the current study, the microarray data from Friis et al (15) was further analyzed in order to identify inflammatory response-associated genes in the ES by constructing a protein-protein interaction (PPI) network, through module analysis and by common gene screening.

\section{Materials and methods}

Microarray data. The microarray data were obtained from the European Bioinformatics Institute database (ebi.ac.uk) with the accession number E-MEXP-3022 (15). The dataset contained three biological replicates of ES plus dura tissues and three biological replicates of pure dura tissues obtained from 10-week-old Lewis inbred rats.

Data normalization and identification of differentially expressed genes (DEG). Raw data were downloaded from the A-AFFY-43-Affymetrix GeneChip Rat Genome 2302.0 (Rat230_2) platform (ebi.ac.uk/arrayexpress/arrays/ A-AFFY-43/?ref=E-MEXP-3022) and preprocessed to normalize and generate probe-level expression data using the Robust Multichip Average algorithm (18) as implemented in the 'affy' package in Bioconductor R (http://www.bioconductor.org/packages/release/bioc/html/affy.html). When multiple probe IDs were matched to the same gene symbol, the average value of expression was determined and the probe ID closest to this value was selected to represent the gene symbol.

DEGs between ES plus dura tissues and pure dura tissues were identified using the Linear Model for Microarray data t-test method (19) from the Bioconductor R package (http://www. bioconductor.org/packages/release/bioc/html/limma.html). Genes were considered differentially expressed at $\mathrm{P}<0.05$ and $\mid \log \mathrm{FC}$ (fold change) $\mid>1$.

To determine whether DEGs have the ability to differentiate between ES plus dura tissues and pure dura tissues, clustering analysis (20) was performed to generate a heat map using the ComplexHeatmap R package (https://github. com/jokergoo/ComplexHeatmap).

PPI network construction. DEGs were mapped into the Search Tool for the Retrieval of Interacting Genes/Proteins 10.0 database (http://string db.org) (21) to obtain PPI pairs. Pairs with PPI score $\geq 0.4$ were retained to construct the PPI network using Cytoscape software 2.8 (www.cytoscape.org) (22).

Crucial nodes within the PPI network were identified by calculating three topological properties using
Table I. Top 20 differentially expressed genes.

\begin{tabular}{|c|c|c|}
\hline Gene & $\log \mathrm{FC}$ & P-value \\
\hline \multicolumn{3}{|l|}{ Upregulated } \\
\hline Ambn & 7.17 & $<0.001$ \\
\hline Itgb6 & 3.90 & $<0.001$ \\
\hline Foxi1 & 4.21 & $<0.001$ \\
\hline $\mathrm{Smr} 3 \mathrm{~b}$ & 4.89 & $<0.001$ \\
\hline Atp6v0a4 & 3.87 & $<0.001$ \\
\hline Ppp1r1b & 3.81 & $<0.001$ \\
\hline Aldh1a1 & 4.60 & $<0.001$ \\
\hline Cwh43 & 3.57 & $<0.001$ \\
\hline Pigr & 4.70 & $<0.001$ \\
\hline Rab17 & 2.23 & $<0.001$ \\
\hline Cldn16 & 4.27 & $<0.001$ \\
\hline Hoxa5 & 4.08 & $<0.001$ \\
\hline Cldn4 & 3.87 & $<0.001$ \\
\hline Perp & 4.03 & $<0.001$ \\
\hline Tbc1d9 & 3.68 & $<0.001$ \\
\hline Pcdh10 & 4.20 & $<0.001$ \\
\hline Pinlyp & 3.60 & $<0.001$ \\
\hline Coch & 3.55 & $<0.001$ \\
\hline Enpp5 & 3.26 & $<0.001$ \\
\hline Fbxo2 & 4.00 & $<0.001$ \\
\hline \multicolumn{3}{|l|}{ Downregulated } \\
\hline Mcpt111 & -3.12 & $<0.001$ \\
\hline Tpsab1 & -3.64 & $<0.001$ \\
\hline LOC102555472 & -2.91 & $<0.001$ \\
\hline Kcne4 & -1.99 & $<0.001$ \\
\hline Cpxm1 & -3.67 & $<0.001$ \\
\hline Alx1 & -3.63 & $<0.001$ \\
\hline Slc5a6 & -1.63 & $<0.001$ \\
\hline Cpxm2 & -1.88 & $<0.001$ \\
\hline Fmod & -1.54 & $<0.001$ \\
\hline Slc6a8 & -1.59 & $<0.001$ \\
\hline Cdkn1c & -1.48 & $<0.001$ \\
\hline RGD1305645 & -1.84 & $<0.001$ \\
\hline Efnb3 & -1.40 & $<0.001$ \\
\hline Aox 1 & -1.76 & $<0.001$ \\
\hline Olfml3 & -1.39 & $<0.001$ \\
\hline Krt33b & -1.94 & $<0.001$ \\
\hline Krt12 & -3.24 & $<0.001$ \\
\hline Elmod1 & -1.73 & $<0.001$ \\
\hline Vnn1 & -1.86 & $<0.001$ \\
\hline Mcpt10 & -2.89 & $<0.001$ \\
\hline
\end{tabular}

FC, fold change.

the CytoNCA plugin (version 2.1.6, parameter: Without weight) in the Cytoscape software (http://apps.cytoscape. org/apps/cytonca) (23), including the degree [the number of interactions per node (protein)] (24), the betweenness (the number of shortest paths that pass through each node) (25) 


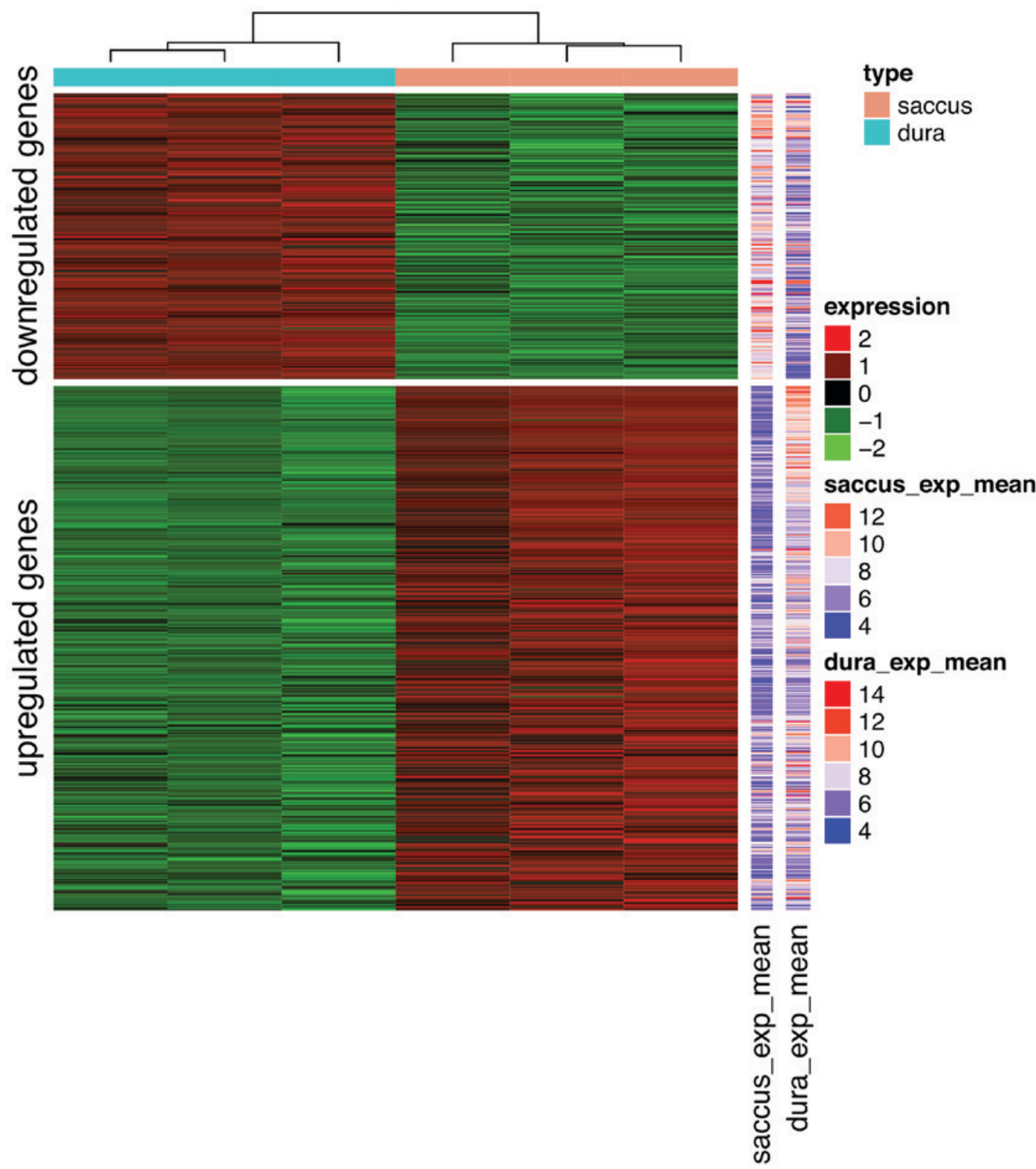

Figure 1. Heat map of differentially expressed genes between endolymphatic sac plus dura tissues and pure dura tissues from rats. Analysis is based on microarray data (accession no. E-MEXP-3022) available from the European Bioinformatics Institute database.

and subgraph centrality (the weighted sum of all closed walks originating from each node) (26).

Functionally associated and densely interconnected modules were extracted from the PPI network using Clustering with Overlapping Neighborhood Expansion (ClusterONE version 1.0; ftp://ftp.mshri.on.ca/pub/BIND/Tools/MCODE) in the Cytoscape software (27). Significant submodules were identified using $\mathrm{P}<0.01$ and nodes $\geq 6$.

Function enrichment analysis. To explain the underlying functions of DEGs, Gene ontology (GO), including the terms of molecular function (MF), biological process (BP) and cellular component (CC), and Kyoto Encyclopedia of Genes and Genomes pathway enrichment analyses were performed using the Database for Annotation, Visualization and Integrated Discovery (DAVID) 6.8 online tool (http://david.abcc.ncifcrf. gov) (28) based on hypergeometric tests. Counts $\geq 2$ and $\mathrm{P}<0.05$ were set as cut-off values.
Comparison with previous literature. To further confirm the key genes in ES for AIED, the results were also compared with previous studies by Møller et al $(16,17)$, which investigated the gene expression profile of ES in humans.

\section{Results}

Identification of DEGs. A total of 612 genes were identified as DEGs between ES plus dura tissues and pure dura tissues based on the set threshold $(\mathrm{P}<0.05$ and $\mid \log \mathrm{FCl}>1)$, including 396 up- and 216 downregulated genes (Table I). Identified DEGs may allow to distinguish ES plus dura tissues from pure dura tissues according to the heat map presented in Fig. 1.

Function enrichment analysis of DEGs. GO enrichment analysis was performed for all DEGs to reveal underlying functions. As a result, 116 (59 BP, $35 \mathrm{CC}$ and $22 \mathrm{MF}$ ) GO terms were enriched for upregulated DEGs, including GO:0007155-cell 


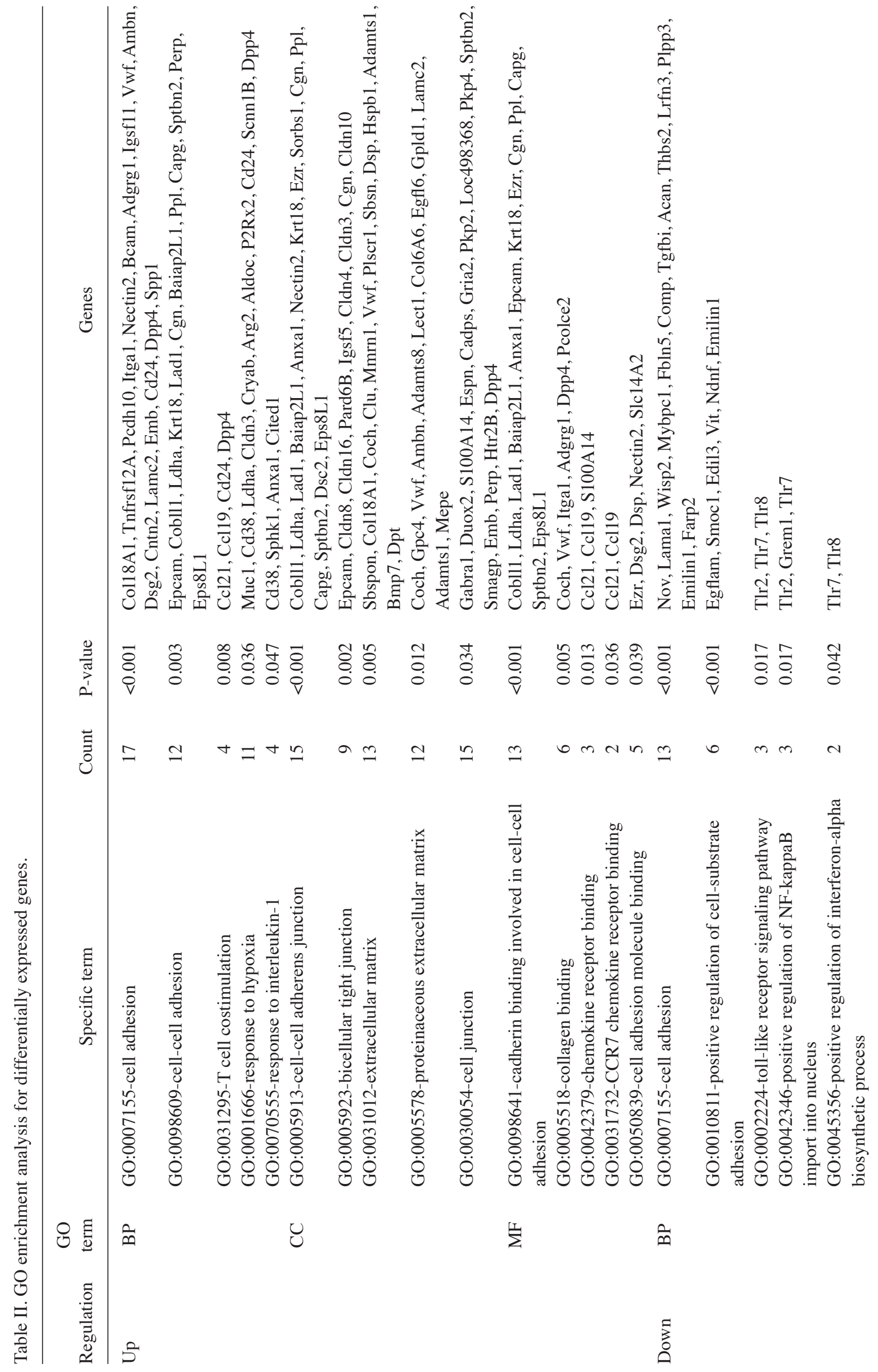




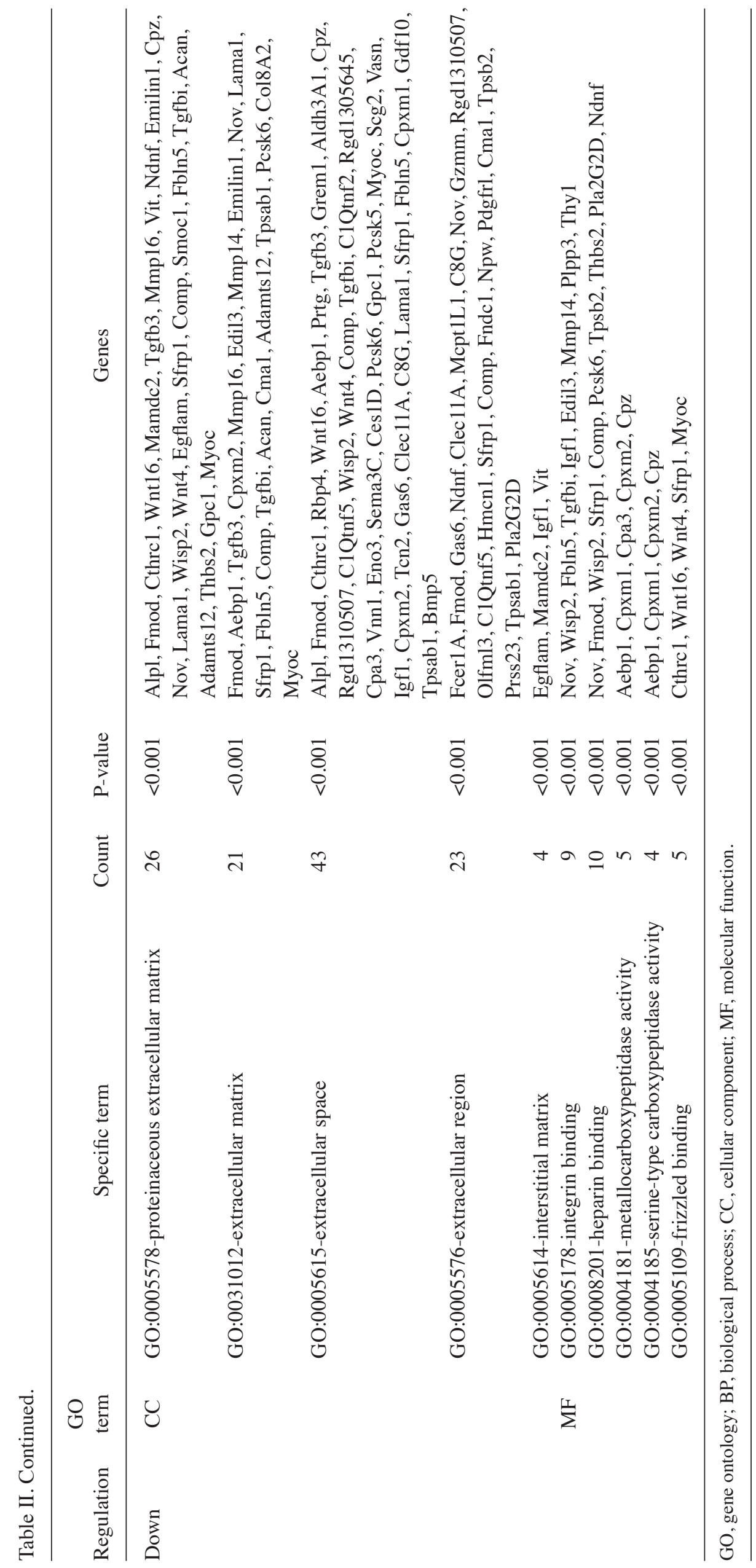


Table III. Kyoto encyclopedia of genes and genomes pathway enrichment analysis for differentially expressed genes.

\begin{tabular}{|c|c|c|c|c|}
\hline Regulation & Term & Count & P-value & Genes \\
\hline \multirow[t]{6}{*}{$\mathrm{Up}$} & rno04530:Tight junction & 11 & $<0.001$ & $\begin{array}{l}\text { Cldn8, Cldn16, Pard6B, Igsf5, Cldn4, Cldn3, Cgn, } \\
\text { Cldn10, Myh14, My19, Llg12 }\end{array}$ \\
\hline & rno04966:Collecting duct acid secretion & 5 & 0.002 & Atp6V1C2, Clcnkb, Atp6V1G3, Atp6V0A4, Car2 \\
\hline & rno04974:Protein digestion and absorption & 8 & 0.002 & $\begin{array}{l}\text { Col18A1, Kcnn4, Col9A3, Col6A6, Mme, Slc1A1, } \\
\text { Kcnq1, Dpp4 }\end{array}$ \\
\hline & $\begin{array}{l}\text { rno05412:Arrhythmogenic right } \\
\text { ventricular cardiomyopathy }\end{array}$ & 7 & 0.003 & Dsg2, Pkp2, Itgb6, Itga1, Dsc2, Dsp, Cacna2D3 \\
\hline & rno04512:ECM-receptor interaction & 7 & 0.009 & Vwf, Lamb3, Col6A6, Itgb6, Itga1, Lamc2, Spp1 \\
\hline & $\begin{array}{l}\text { rno04670:Leukocyte transendothelial } \\
\text { migration }\end{array}$ & 7 & 0.034 & Cldn8, Cldn16, Ezr, Cldn4, Cldn3, Cldn10, My19 \\
\hline \multirow[t]{5}{*}{ Down } & rno05205:Proteoglycans in cancer & 7 & 0.008 & Wnt16, Wnt4, Tlr2, Igf1, Fzd2, Gpc1, Twist1 \\
\hline & rno04916:Melanogenesis & 5 & 0.011 & Wnt16, Wnt4, Adcy8, Creb3L1, Fzd2 \\
\hline & rno05144:Malaria & 4 & 0.015 & Comp, Tlr2, Tgfb3, Thbs2 \\
\hline & rno04977:Vitamin digestion and absorption & 3 & 0.016 & Slc5A6, Tcn2, Slc19A2 \\
\hline & rno05200:Pathways in cancer & 9 & 0.022 & Lama1, Wnt16, Wnt4, Adcy8, Rasgrp2, Tgfb3, Skp2, \\
\hline \multicolumn{5}{|c|}{ Igf1, Fzd2 } \\
\hline & rno05414:Dilated cardiomyopathy & 4 & 0.037 & Sgcg, Adcy8, Tgfb3, Igf1 \\
\hline & rno04390:Hippo signaling pathway & 5 & 0.044 & Wnt16, Wnt4, Tgfb3, Fzd2, Bmp5 \\
\hline
\end{tabular}

Table IV. Top 20 hub genes presented by topological property extracted from the protein-protein interaction network.

\begin{tabular}{clll}
\hline & \multicolumn{3}{c}{ Gene (value) } \\
\cline { 2 - 4 } Rank & \multicolumn{1}{c}{ Degree $^{\mathrm{a}}$} & \multicolumn{1}{c}{ Subgraph $^{\mathrm{b}}$} & Betweenness $^{\mathrm{c}}$ \\
\hline \multirow{2}{*}{1} & Ripk4 (47) & Ripk4 $(11,351)$ & Ripk4 $(28,108)$ \\
2 & Dpyd (31) & Dpyd $(8,498)$ & Fos $(15,278)$ \\
3 & Actg2 (31) & Actg2 $(6,488)$ & Dpyd $(10,661)$ \\
4 & Fos (25) & Aldh3a1 $(6,317)$ & Agt $(10,644)$ \\
5 & Pcsk5 (24) & Aldh1a3 $(6,296)$ & Calb1 $(8,620)$ \\
6 & Aldh1a1 (23) & Aldh1a1 $(6,260)$ & Spp1 $(7,876)$ \\
7 & Agt (23) & Pcsk5 $(3,030)$ & Epcam $(7,590)$ \\
8 & Aldh3a1 (22) & Gad1 $(2,611)$ & Actg2 $(6,459)$ \\
9 & Aldh1a3 (22) & Calb1 $(2,598)$ & Myo5c $(6,141)$ \\
10 & Calb1 (22) & Pcsk6 $(2,557)$ & Eno3 $(5,163)$ \\
11 & Pcsk6 (22) & Rasd1 $(2,524)$ & Txndc5 $(4,214)$ \\
12 & Myo5c (21) & Myo5c $(2,491)$ & Cd24 $(4,135)$ \\
13 & Gad1 (16) & Eno3 $(2,170)$ & Krt8 $(4,077)$ \\
14 & Eno3 (16) & Myo5b $(2,134)$ & Aldh1a1 $(3,840)$ \\
15 & Myo5b (16) & Rdh10 $(1,939)$ & Pcsk5 $(3,802)$ \\
16 & Rasd1 (15) & Bdh2 $(1,906)$ & Pcsk6 $(3,788)$ \\
17 & Itga1 (14) & Hpgd $(1,906)$ & Itga1 $(3,674)$ \\
18 & Igf1 (14) & Dhrs7c $(1,906)$ & Gad1 $(3,617)$ \\
19 & Itgb6 (14) & Fos $(1,883)$ & Hspa1a $(3,589)$ \\
20 & Hspa1a, Spp1 (13) & Cndp1 $(1,832)$ & Itgb6 $(3,555)$ \\
& & &
\end{tabular}

${ }^{\mathrm{a}}$ The number of interactions per node (protein). ${ }^{\mathrm{b}}$ Subgraph centrality, the weighted sum of all closed walks originating from each node. 'Betweenness, the number of shortest paths that pass through each node.
Table V. Genes associated with immune response identified in rats and humans, obtained from a comparison with a study by Møller et al (16).

\begin{tabular}{lrr}
\hline Gene & $\operatorname{logFC}$ & P-value \\
\hline Tlr7 & -1.15 & 0.003 \\
Tlr2 & -1.23 & 0.003 \\
Tlr8 & -1.40 & $<0.001$ \\
Lpo & 2.02 & 0.004 \\
Gzmm & -1.48 & $<0.001$ \\
Muc1 & 3.28 & $<0.001$ \\
\hline
\end{tabular}

FC, fold change; Tlr, toll-like receptor; Lpo, lactoperoxidase; Gzmm, granzyme m; Muc, mucin.

adhesion, with $\alpha 5$-integrin (Itga1) and secreted phosphoprotein 1 (Spp1); GO:0031295-T cell co-stimulation, with Ccl21 and Ccl19; and GO:0070555-response to IL-1, with Ccl21 and Ccl19 (Table II). For downregulated DEGs, 77 (including $48 \mathrm{BP}, 11 \mathrm{CC}$, and $18 \mathrm{MF}$ ) GO terms were enriched. In line with the upregulated DEGs, the majority of downregulated DEGs was associated with GO:0007155-cell adhesion, with transforming growth factor (Tgf)- $\beta \mathrm{i}$; GO:0002224-toll-like receptor signaling pathway, with Tlr2, Tlr7 and Tlr8; and GO:0042346-positive regulation of nuclear factor (NF)- $\kappa \mathrm{B}$ import into the nucleus, with Tlr2 and Tlr7 (Table II).

Pathway analyses were also performed using DAVID software. Upregulated DEGs were predicted to participate in six pathways, including rno04530: Tight junction, with claudin (Cldn)-4; rno04512: Extracellular matrix (ECM)-receptor 




Figure 2. Protein-protein interaction network created with differentially expressed genes associated to autoimmune inner ear diseases. Red and green nodes represent upregulated and downregulated genes, respectively.

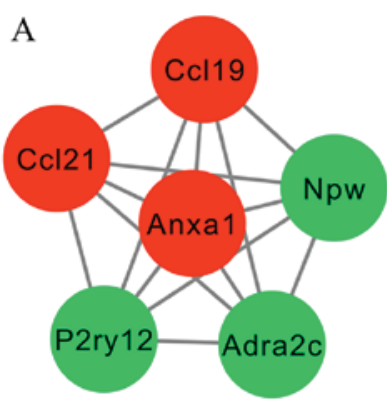

$\mathrm{D}$

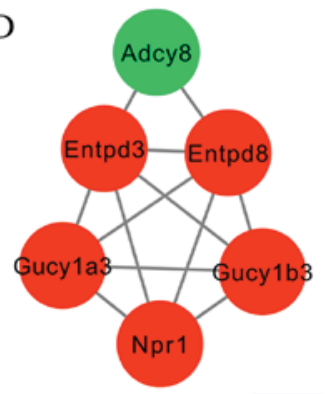

$\mathrm{B}$

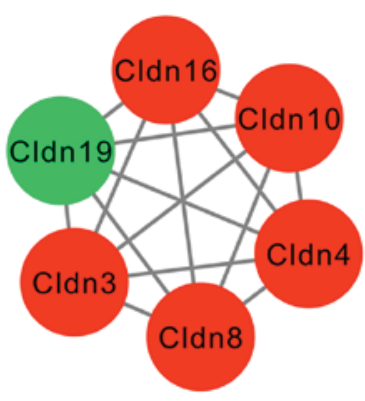

$\mathrm{C}$

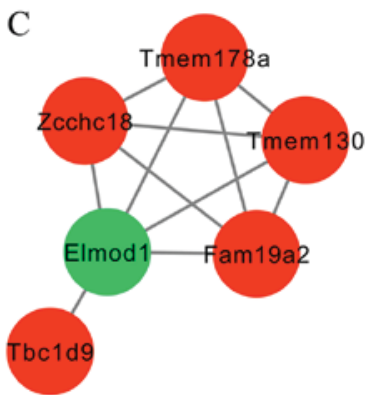

E

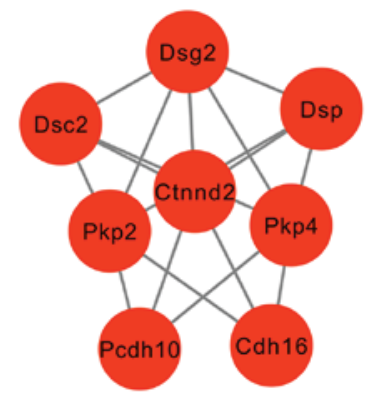

Figure 3. Module analysis for differentially expressed genes that were extracted from protein-protein interaction network. Module (A) 1 , (B) 2 , (C) 3 , (D) 4 and (E) 5. Red and green nodes represent upregulated and downregulated genes, respectively.

interaction, with Itga1 and Spp1; and rno04670: Leukocyte transendothelial migration, with Cldn4 (Table III). Downregulated DEGs were enriched in seven pathways, including inflammation-associated diseases, rno05144: Malaria, with Tgf- $\beta 3$ and Tlr2 (Table III).

PPI network construction. Following the mapping of DEGs into protein interactions pairs, a PPI network was constructed, including 338 nodes and 777 edges (interaction relationships; Fig. 2). Key nodes in the PPI network were screened by calculating the degree, betweenness and subgraph centrality. Ripk4, Dpyd, Actg2, Fos, Pcsk5, Aldh1a1, Calb1, Pcsk6, Myo5c, Gad1 and Eno3 were in the top 20 genes of the three topological characteristics, but were not determined to be enriched in adhesion or immune pathways. Itga1 and Spp1 were enriched in adhesion or immune pathways as described above, they 

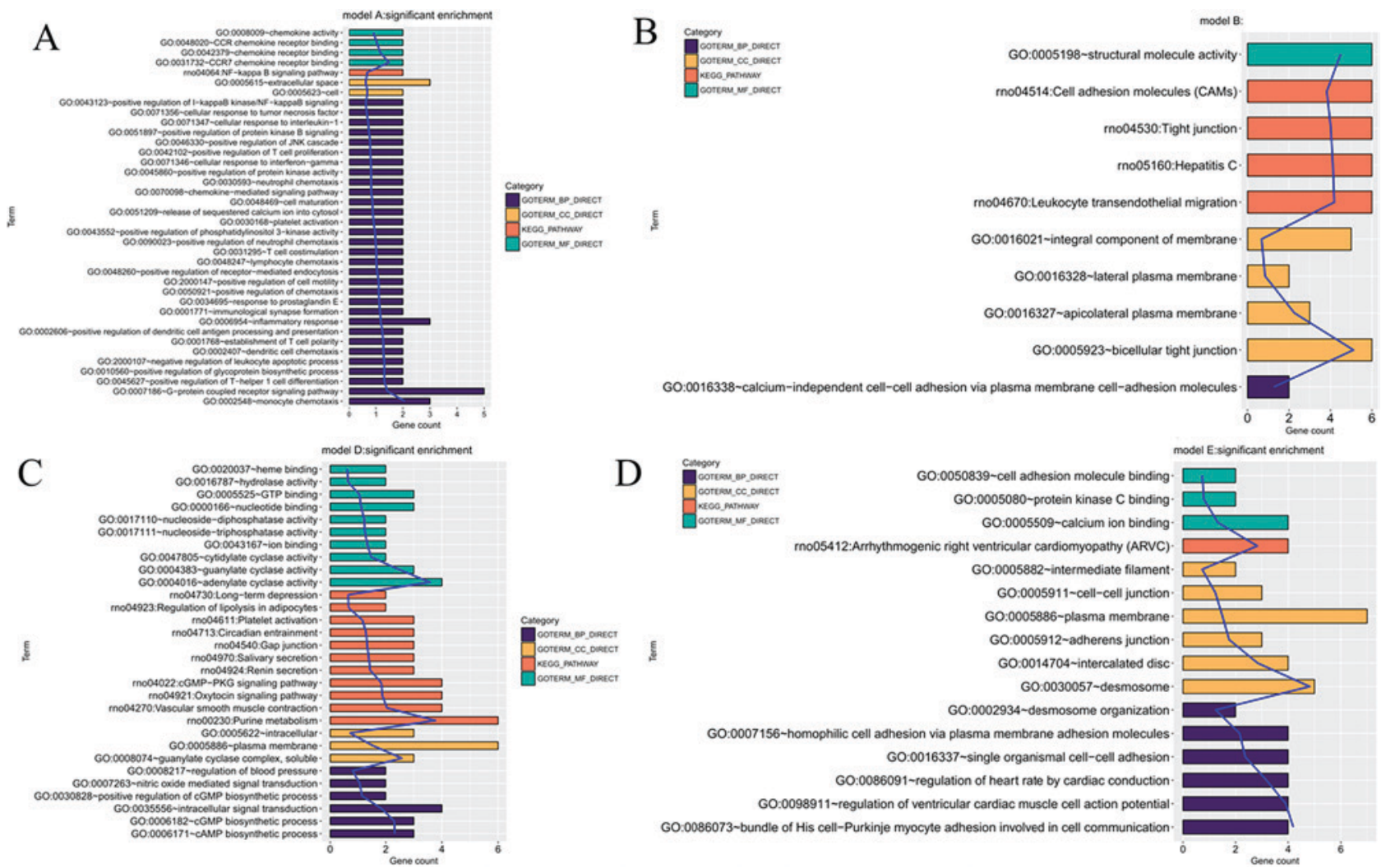

Figure 4. Function enrichment for modules. Module (A) 1, (B) 2, (C) 4 and (D) 5. Module 3 is not included as only one GO term was enriched. GO, gene ontology; MF, molecular function; BP, biological process; CC, cellular component; KEGG, Kyoto encyclopedia of genes and genomes.

were also found to be crucial according to their degree and/or betweenness centrality (Table IV).

Following ClusterONE analysis, 5 significant submodules were created (Fig. 3). Function enrichment analysis revealed that the genes participating in module 1 were closely associated with inflammation, including $\mathrm{Ccl} 21$ and $\mathrm{Ccl} 19$, whereas the genes in module 2 and 5 were significantly enriched in cell adhesion pathways, including Cldn4 (Fig. 4). In module 3, only one GO term, GO:0005096-GTPase activator activity, was enriched and module 3 is not presented in Fig. 4.

Identification of common genes in rats and humans by literature comparison. Møller et al (16) performed a study to investigate gene expression of the human ES using adjacent dura mater as the control. The authors identified that ES was a significant immunological entity within the inner ear. To screen for immune response-associated genes in the ES, the DEGs from rats from the current study were compared with human DEGs, including Tlr1, Tlr2, Tlr3, Tlr4, Tlr7, Fc fragment of $\mathrm{IgA}$ and $\mathrm{IgM}$ receptor, $\mathrm{C}-\mathrm{C}$ chemokine receptor 7, C-X-C chemokine (CXC) ligand $17, \mathrm{CXC}$ receptor 3 , $\alpha$-interferon, IL-17, $\beta$-defensins, lactotransferrin precursor, lactoperoxidase (Lpo), perforin 1, histatin, granzyme (Gzm) and mucins (Mucs). A total of 6 common genes were identified, including Tlr7, Tlr2, Tlr8, Lpo, Gzmm and Muc1 (Table V). Separate function analysis of these genes was performed. Tlr7, Tlr2, Tlr8 and Gzmm were enriched in immune or inflammatory response-associated GO terms or pathways (Fig. 5). No enrichment was observed for Lpo and Muc1, indicating Tlr7, Tlr2, Tlr8 and Gzmm were of particular importance.

\section{Discussion}

Compared with the previous study from Friis et al (15), a less restrictive threshold value $[\mathrm{P}<0.05$ and $\mid \log \mathrm{FC}$ (fold change) $\mid>1$ vs. false discovery rate $<0.1]$ was adopted for screening of DEGs in the ES in the current study. As a result, 619 DEGs were identified compared with 463 DEGs reported by Friis et al (15). Subsequently, DEGs were subjected to a PPI network construction, module analysis, function analysis and a comparison with the results reported by Møller et al (16), none of which was included in the work presented by Friis et al (15). The results of the current study may provide a more solid base compared with previous work and novel conclusions were obtained. In the present study, a series of inflammatory response-associated genes were screened. The hub roles of Itga1, Cd24 and Spp1 were identified by calculating three topological properties of the PPI network. Ccl21, Ccl19 and Cldn4 were demonstrated to be crucial following significant module analysis according to the corresponding threshold, which revealed they were enriched in inflammation pathways. Tlr7, Tlr2, Gzmm and Tlr8 were identified as common genes associated with inflammatory responses in rat and human ES (16). Abnormal expressions of the aforementioned inflammatory-associated genes may describe the underlying mechanism for the development of AIED.

Ccl21, Ccl19, Tlr7, Tlr2 and Tlr8 are known inflammatory-associated genes (29-33,34-36), making their presence in ES and association with AIED plausible. It has previously been reported that $\mathrm{Ccl} 19$ and $\mathrm{Ccl} 21$ were significantly induced in several autoimmune diseases, including 


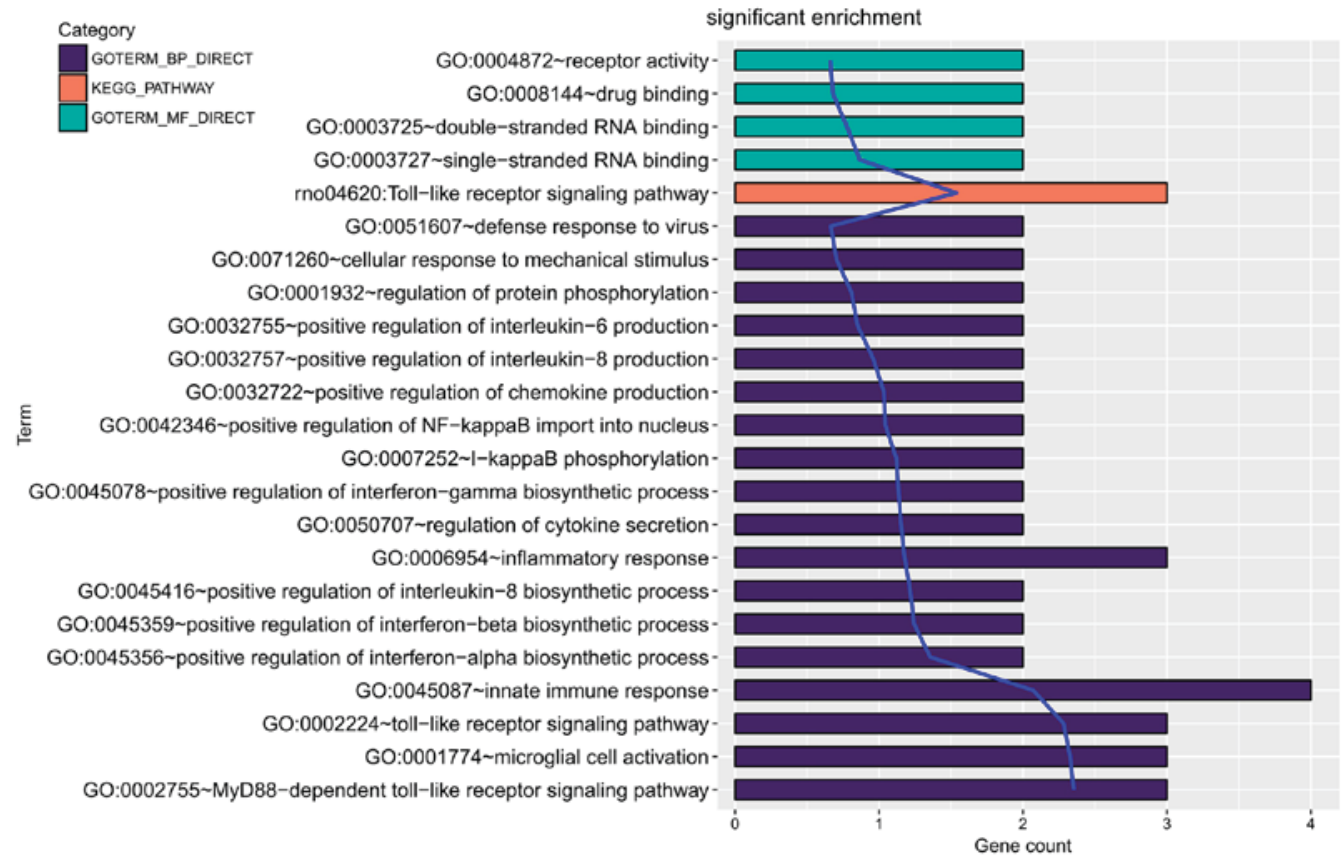

Figure 5. Function enrichment for six immune or inflammatory response-associated genes common in rats and human. GO, gene ontology; MF, molecular function; BP, biological process; KEGG, Kyoto encyclopedia of genes and genomes.

rheumatoid arthritis (29), autoimmune encephalomyelitis (30) and multiple sclerosis (31) which have been diagnosed in $\leq 1 / 3$ of patients with AIED $(32,33)$. High expressions of Ccl19 and $\mathrm{Ccl} 21$ were demonstrated to initiate specific immune responses by co-stimulating the expansion of $\mathrm{CD} 4^{+}$and $\mathrm{CD} 8^{+}$ T cells (32), inducing Th1 (37) and macrophage polarization to secrete inflammatory cytokines (including IL-8) (29), and mediating antigen presenting cell trafficking (including dendritic cells) (38). A combination of the continuous local antigenic stimulation and the maintenance of chronic inflammation ultimately lead to the development of AIED.

Tlrs are associated with the activation of the innate immune system by recognizing pathogen-associated molecular patterns (39). Proinflammatory effects have been described for Tlrs previously (34), whereas other studies implied protective effects of activated Tlr2, Tlr7 and Tlr8 in mouse models of asthma $(35,36,40)$. Treatment with Tlr7/8 agonist, resiquimod (RSQ), was reported to reduce allergen-induced airway reactivity and inflammation (35). The underlying mechanism is associated with the induction of NF-E2-related factor 2 and copper/zinc superoxide dismutase (35). The suppressive effect of RSQ in asthma is further dependent on the induction of IL-27 and is associated with enhanced expression of the immunomodulatory molecule B7-homolog 1 on pulmonary antigen presenting cells (36). Pam3Cys, a Tlr2 agonist treatment, has been demonstrated to cause significant reductions of eosinophils and increase numbers of regulatory $\mathrm{T}$ cells (Tregs) in lung infiltrates, resulting in long-term protection against manifestation of allergic asthma (40). Previous studies revealed that induction of the generation of antigen specific cluster of differentiation $(\mathrm{CD}) 4^{+} \mathrm{CD} 25^{+}$forkhead box P ${ }^{+}$Tregs by adipose tissue-derived stem cells significantly improved hearing function and protected inner hair cells from loss in previous AIED models $(41,42)$. The current study speculated that Tlr2 and Tlr7/8 agonist may be potential agents for the treatment of AIED, which was supported by the observed downregulation of Tlr7, Tlr2 and Tlr8 in ES.

Gzms comprise a family of serine protease $(a, b, h, k$ and $\mathrm{m}$ ) that is constitutively and abundantly expressed in innate effector natural killer cells to eliminate virus-infected and tumor cells to prevent inflammation $(43,44)$. Mice lacking Gzms exhibited a reduced immune tolerance and increased inflammation levels through a type I interferon-dependent pathway, leading to the development of autoimmune diseases, including diabetes $(43,45)$ and ulcerative colitis $(46)$. These two autoimmune diseases were further associated with SNHL $(47,48)$. Gzmm was proposed to be downregulated in AIED, which was in accordance with the results of the current study.

The ECM is a non-cellular component in all tissues and organs and adherens junctions between cells, and are essential for the maintenance of the structural and functional integrity of organs (49). Disruption of ECM and adherens junctions may contribute to the destruction of the epithelial barrier and favor the interaction of leukocytes with endothelial cells, promoting the development of inflammation-associated diseases (50), including AIED. Cai et al (51) previously reported that the expression of ECM gene Itga5 in the basal sections of the sensory epithelium in the cochlea was positively correlated with greater hearing loss as a result to exposure to an intense noise for $2 \mathrm{~h}$. Endogenous overexpression of another ECM gene, SPP1, induces Ccl5 and matrix metalloproteinase-2 activation that contributes to proinflammatory events (52). Lee et al (50) recently demonstrated that the level of Cldn4, coding for a tight junction protein, was increased in blood and plasma of asthmatic patients and lung tissues from the model mice, accompanied with significant increases in tight junction disruptions. A Cldn4 knockdown by small interfering RNA transfection significantly increased inflammatory cytokine 
expression (50). In accordance with these findings, SPP1, Itga1 and Cldn4 were upregulated in rats ES, suggesting these genes may be underlying targets for the treatment of AIED.

In conclusion, the present study revealed a number of important inflammatory-associated genes, including Tlr2, Tlr7, Tlr8, Gzmm, Itga1, Spp1 and Cldn4, in ES. Abnormal expression of these genes may describe the underlying mechanism for the development of AIED. Further studies with an AIED animal model are required to confirm these conclusions.

\section{Acknowledgements}

Not applicable.

\section{Funding}

The present study was supported by National Natural Science Foundation of China (grant nos. 81371084 and 81570924).

\section{Availability of data and materials}

The E-MEXP-3022 microarray datasets analyzed during the current study are available in the European Bioinformatics Institute database repository (ebi.ac.uk).

\section{Author's contributions}

JZ and AX participated in the design of the study. JZ and NW performed the bioinformatics analysis. JZ, NW and AX contributed to the acquisition and interpretation of the data. JZ drafted the manuscript. AX revised the manuscript. All authors read and approved the final manuscript.

\section{Ethics approval and consent to participate}

Not applicable.

\section{Patient consent for publication}

Not applicable.

\section{Competing interests}

The authors declare that they have no competing interests.

\section{References}

1. Matsuoka AJ and Harris JP: Autoimmune inner ear disease: A retrospective review of forty-seven patients. Audiol Neurootol 18 228-239, 2013

2. Huang NC and Sataloff RT: Autoimmune inner ear disease in children. Otol Neurotol 32: 213-216, 2011.

3. Dougherty W, Thatayatikom A and Bush ML: Paediatric autoimmune inner ear disease: A case series. Audiol Med 13: 32-39, 2015.

4. Chen J, Liang J, Ou J and Cai W: Mental health in adults with sudden sensorineural hearing loss: An assessment of depressive symptoms and its correlates. J Psychosom Res 75: 72-74, 2013.

5. Carlsson PI, Hall M, Lind KJ and Danermark B: Quality of life, psychosocial consequences, and audiological rehabilitation after sudden sensorineural hearing loss. Int J Audiol 50: 139-144, 2011.

6. Niparko JK, Wang NY, Rauch SD, Russell GB, Espeland MA, Pierce JJ, Bowditch S, Masuda A, Gulya AJ, Gantz BJ, et al: Serial audiometry in a clinical trial of AIED treatment. Otol Neurotol 26: 908-917, 2005.
7. Alexander TH, Weisman MH, Derebery JM, Espeland MA, Gantz BJ, Gulya AJ, Hammerschlag PE, Hannley M, Hughes GB, Moscicki R, et al: Safety of high-dose corticosteroids for the treatment of autoimmune inner ear disease. Otol Neurotol 30: $443-448,2009$.

8. Kanaya T, Kamito T, Shirato M and Unno T: Steroid dependent sensorineural hearing loss in a patient with bilateral meniere's disease. Practica Oto-Rhino-Laryngologica 85: 191-196, 1992.

9. Goodall AF and Siddiq MA: Current understanding of the pathogenesis of autoimmune inner ear disease: A review. Clin Otolaryngol 40: 412-419, 2015.

10. Takahashi M and Harris JP: Anatomic distribution and localization of immunocompetent cells in normal mouse endolymphatic sac. Acta Otolaryngol 106: 409-416, 1988.

11. Gopen Q, Keithley EM and Harris JP: Mechanisms underlying autoimmune inner ear disease. Drug Discov Today Dis Mech 3: 137-142, 2006. https://doi.org/10.1016/j.ddmec.2006.02.006.

12. Satoh H, Firestein GS, Billings PB, Harris JP and Keithley EM: Proinflammatory cytokine expression in the endolymphatic sac during inner ear inflammation. J Assoc Res Otolaryngol 4: 139-147, 2003.

13. Pathak S, Stern C and Vambutas A: N-Acetylcysteine attenuates tumor necrosis factor alpha levels in autoimmune inner ear disease patients. Immunol Res 63: 236-245, 2015.

14. Vambutas A, Lesser M, Mullooly V, Pathak S, Zahtz G, Rosen L and Goldofsky E: Early efficacy trial of anakinra in corticosteroid-resistant autoimmune inner ear disease. J Clin Invest 124: 4115-4122, 2014.

15. Friis M, Martinbertelsen T, Friishansen L, Winther O, Henao R, Sørensen MS and Qvortrup K: Gene expression of the endolymphatic sac. Acta Otolaryngol 131: 1257-1263, 2011

16. MøllerMN,KirkebyS,Vikeså J,NielsenFCand Cayé-ThomasenP Gene expression demonstrates an immunological capacity of the human endolymphatic sac. Laryngoscope 125: E269-E275, 2015.

17. Møller MN, Kirkeby S, Vikeså J, Nielsen FC and Cayé-Thomasen P: Neuronal fibers and neurotransmitter receptor expression in the human endolymphatic sac. Otol Neurotol 38: 765-773, 2017.

18. Irizarry RA, Bolstad BM, Collin F, Cope LM, Hobbs B and Speed TP: Summaries of Affymetrix GeneChip probe level data. Nucleic Acids Res 31: e15, 2003.

19. Ritchie ME, Phipson B, Wu D, Hu Y, Law CW, Shi W and Smyth GK: limma powers differential expression analyses for RNA-sequencing and microarray studies. Nucleic Acids Res 43: e47, 2015.

20. Gu Z, Eils R and Schlesner M: Complex heatmaps reveal patterns and correlations in multidimensional genomic data. Bioinformatics 32: 2847-2849, 2016.

21. Szklarczyk D, Franceschini A, Wyder S, Forslund K, Heller D, Huerta-Cepas J, Simonovic M, Roth A, Santos A, Tsafou KP, et al: STRING v10: Protein-protein interaction networks, integrated over the tree of life. Nucleic Acids Res 43: D447-D452, 2015.

22. Kohl M, Wiese S and Warscheid B: Cytoscape: Software for visualization and analysis of biological networks. Methods Mol Biol 696: 291-303, 2011.

23. Tang Y, Li M, Wang J, Pan Y and Wu FX: CytoNCA: A cytoscape plugin for centrality analysis and evaluation of protein interaction networks. Biosystems 127: 67-72, 2015.

24. Rito T, Deane CM and Reinert G: The importance of age and high degree, in protein-protein interaction networks. J Comput Biol 19: 785-795, 2012.

25. Wang H, Hernandez JM and Van MP: Betweenness centrality in a weighted network. Phys Rev E Stat Nonlin Soft Matter Phys 77: 046105, 2008.

26. Estrada E and Rodríguez-Velázquez JA: Subgraph centrality in complex networks. Phys Rev E Stat Nonlin Soft Matter Phys 71: $056103,2005$.

27. Nepusz T, Yu H and Paccanaro A: Detecting overlapping protein complexes in protein-protein interaction networks. Nat Methods 9: 471-472, 2012.

28. Dennis G Jr, Sherman BT, Hosack DA, Yang J, Gao W, Lane HC and Lempicki RA: DAVID: Database for annotation, visualization, and integrated discovery. Genome Biol 4: P3, 2003.

29. Pickens SR, Chamberlain ND, Volin MV, Pope RM, Mandelin AM II and Shahrara S: Characterization of CCL19 and CCL21 in rheumatoid arthritis. Arthritis Rheum 63: 914-922, 2011.

30. Columbacabezas S, Serafini B, Ambrosini E and Aloisi F: Lymphoid chemokines CCL19 and CCL21 are expressed in the central nervous system during experimental autoimmune encephalomyelitis: Implications for the maintenance of chronic neuroinflammation. Brain Pathol 13: 38-51, 2003. 
31. Bielecki B, Jatczak-Pawlik I, Wolinski P, Bednarek A and Glabinski A: Central nervous system and peripheral expression of CCL19, CCL21 and their receptor CCR7 in experimental model of multiple sclerosis. Arch Immunol Ther Exp (Warsz) 63: 367-376, 2015.

32. Morovic Vergles J, Radic M, Kovacic J and Salamon L: Successful use of adalimumab for treating rheumatoid arthritis with autoimmune sensorineural hearing loss: Two birds with one stone. J Rheumatol 37: 1080-1081, 2010.

33. Tanaka M and Tanaka K: Sudden hearing loss as the initial symptom in Japanese patients with multiple sclerosis and seropositive neuromyelitis optica spectrum disorders. J Neuroimmunol 298: 16-18, 2016.

34. Jiménezdalmaroni MJ, Gerswhin ME and Adamopoulos IE: The critical role of toll-like receptors-from microbial recognition to autoimmunity: A comprehensive review. Autoimmun Rev 15: $1-8,2016$.

35. Nadeem A, Siddiqui N, Alharbi NO, Alharbi MM and Ahmad SF: TLR-7 agonist attenuates airway reactivity and inflammation through Nrf2-mediated antioxidant protection in a murine model of allergic asthma. Int J Biochem Cell Biol 73: 53-62, 2016.

36. Daluge K, Jirmo AC, Busse M and Hansen G: TLR 7/8 agonist resiquimod alleviates murine allergic asthma through IL-27 production and up regulation of $\mathrm{B} 7-\mathrm{H} 1$ on antigen presenting cells. Eur Respir J 46: PA1896, 2015.

37. Flanagan K, Moroziewicz D, Kwak H, Hörig H and Kaufman HL: The lymphoid chemokine CCL21 costimulates naive T cell expansion and Th1 polarization of non-regulatory $\mathrm{CD}^{+}{ }^{+} \mathrm{T}$ cells Cell Immunol 231: 75-84, 2004

38. Hua J,Stevenson W,DohlmanT, CalcagnoN,PirmadjidN,SadraiZ, Chauhan S, Saban D and Dana R: The CCR7-CCL19/CCL21 axis mediates enhanced antigen-presenting cell trafficking in high-risk corneal transplantation. Invest Ophthalmol Vis Sci 54: 1289,2013

39. Underhill DM: Collaboration between the innate immune receptors dectin-1, TLRs, and Nods. Immunol Rev 219: 75-87, 2010.

40. Nawijn MC, Motta AC, Gras R, Shirinbak S, Maazi H and van Oosterhout AJ: TLR-2 activation induces regulatory T cells and long-term suppression of asthma manifestations in mice. Plos One 8: e55307, 2013.

41. Yoo TJ, Du X and Zhou B: The paracrine effect of mesenchymal human stem cells restored hearing in $\beta$-tubulin induced autoimmune sensorineural hearing loss. Hear Res 330: 57-61, 2015.

42. Zhou Y, Yuan J, Zhou B, Lee AJ, Lee AJ, Ghawji M Jr and Yoo TJ: The therapeutic efficacy of human adipose tissue-derived mesenchymal stem cells on experimental autoimmune hearing loss in mice. Immunology 133: 133-140, 2011.
43. García-Laorden MI, Stroo I, Blok DC, Florquin S, Medema JP, de Vos AF and van der Poll T: Granzymes A and B regulate the local inflammatory response during Klebsiella pneumoniae Pneumonia. J Innate Immun 8: 258-268, 2016.

44. Walch M, Dotiwala F, Mulik S, Thiery J, Kirchhausen T, Clayberger C, Krensky AM, Martinvalet D and Lieberman J: Cytotoxic cells kill intracellular bacteria through Granulysin-mediated delivery of Granzymes. Cell 157: $1309-1323,2014$

45. Mollah ZU, Quah HS, Graham KL, Jhala G, Krishnamurthy B Francisca MDJ, Chee J, Trivedi PM, Pappas EG, Mackin L, et al: Granzyme A-deficiency breaks immune tolerance and promotes autoimmune diabetes through a type I interferon-dependent pathway. Diabetes 66: 3041-3050, 2017.

46. Souza-Fonseca-Guimaraes F, Krasnova Y, Putoczki T, Miles K, Macdonald KP, Town L, Shi W, Gobe GC, Mcdade L, Mielke LA, et al: Granzyme M has a critical role in providing innate immune protection in ulcerative colitis. Cell Death Dis 7: e2302, 2016.

47. Casella G, Corbetta D, Zolezzi M, Di Bella C, Villanacci V, Salemme M, Milanesi U, Antonelli E, Baldini V and Bassotti G: Symptomatic sensorineural hearing loss in patients with ulcerative colitis. Tech Coloproctol 19: 729-731, 2015.

48. Lin CF, Lee KJ, Yu SS and Lin YS: Effect of comorbid diabetes and hypercholesterolemia on the prognosis of idiopathic sudden sensorineural hearing loss. Laryngoscope 126: 142-149, 2016.

49. Burridge K, Fath K, Kelly T, Nuckolls G and Turner C: Focal Adhesions: Transmembrane junctions between the extracellular matrix and the cytoskeleton. Annu Rev Cell Biol 4: 487-525, 1988.

50. Lee PH, Kim BG, Lee SH, Lee JH, Park SW, Kim DJ, Park CS, Leikauf GD and Jang AS: Alteration in Claudin-4 contributes to airway inflammation and responsiveness in asthma. Allergy Asthma Immunol Res 10: 25-33, 2018.

51. Cai Q, Patel M, Coling D and Hu BH: Transcriptional changes in adhesion-related genes are site-specific during noise-induced cochlear pathogenesis. Neurobiol Dis 45: 723-732, 2012.

52. Caballero EP, Santamaría MH and Corral RS: Endogenous osteopontin induces myocardial CCL5 and MMP-2 activation that contributes to inflammation and cardiac remodeling in a mouse model of chronic Chagas heart disease. Biochim Biophys Acta 1864: 11-23, 2017.

This work is licensed under a Creative Commons

Attribution-NonCommercial-NoDerivatives 4.0

International (CC BY-NC-ND 4.0) License. 